\title{
Electric-double-layer potential distribution in multiple-layer immiscible electrolytes
}

\author{
Siddhartha Das ${ }^{1}$ and Steffen Hardt ${ }^{2}$ \\ ${ }^{1}$ Physics of Fluids Group and J. M. Burgers Centre for Fluid Dynamics, University of Twente, P.O. Box 217, \\ NL-7500 AE Enschede, The Netherlands \\ ${ }^{2}$ Center of Smart Interfaces, Technische Universität Darmstadt, Petersenstrasse 32, D-64287 Darmstadt, Germany
}

(Received 28 June 2011; revised manuscript received 25 July 2011; published 23 August 2011)

\begin{abstract}
In this Brief Report, we calculate the electric-double-layer (EDL) electrostatic potential in a system of several layers of immiscible electrolytes. Verwey-Niessen theory predicts that at the interface between two immiscible electrolytes back-to-back EDLs are formed. The present analysis extends this idea to the case where the immiscible liquids are contained inside a domain with given electrostatic potentials at its boundaries, where the thickness of the individual liquid layer can be comparable to the EDL thickness. Such a system gives rise to a situation where the overall EDL electrostatic potential in the system is dictated by the competitive influences of the boundary-induced effects and the effects induced by the jump in the ion-solvent interaction potential at the liquid-liquid interfaces. Invoking Debye-Hückel linearization, we derive an analytical result for the EDL electrostatic potential for two immiscible electrolyte layers, and extend it for a general system of $N$ such immiscible electrolyte layers. We demonstrate that, depending upon the nature of the interfacial ion-solvent interaction potential jump, the overall EDL potential may manifest a strong influence of the boundary conditions or may invert the influence of the boundary conditions. Effects such as a variation of the ratio of the permittivity or the thickness of the liquids also dictate the overall potential profiles.
\end{abstract}

DOI: 10.1103/PhysRevE.84.022502

PACS number(s): $82.45 . \mathrm{Gj}$

Ionic distributions at the interface between two immiscible electrolytes have been widely studied for several decades. At such interfaces, back-to-back electric double layers (EDLs) develop — as was first analyzed by Verwey and Niessen [1] based on Gouy-Chapman theory [2,3]. Over the years, there have been several other studies extending Verwey-Niessen theory to include effects like ion adsorption at the interface [4,5], a finite width of the interface [6,7], or ionic-molecular interactions [8], etc.

In the present Brief Report, we address the problem of electric-double-layer (EDL) potential distribution in a system of immiscible electrolytes with given potentials at the domain boundaries, where the EDL thicknesses can be of the same order as the thicknesses of the electrolyte layers. The description is based on analyzing the free energy of the electrolyte system, where the effects of variation in the solvent composition must be accounted for by an appropriate variation in the chemical potential of the ions in contact with a particular solvent. Tsori and Liebler [9] studied a similar problem where they described the free energy of a two-component ion-containing miscible solvent. However, their analysis did not describe the effect of the liquid layer thickness being of the order of the EDL thickness, or the consequence of the interfacial jump in the ionic chemical potential. In this Brief Report, we shall invoke a similar model [9], further simplified to account for immiscible solvents. The central result of this Brief Report is that, under certain conditions, the jump in the ion-solvent interaction potential can be so severe that it may augment the boundary effects to induce an even enhanced (as compared to that at the wall) potential in the bulk, or may nullify the boundary effects to such an extent as to enforce an electrostatic potential of opposite sign (as compared to the boundary) in almost the entire domain.

Among others, the model developed in this Brief Report is relevant for all situations in which a thin electrolyte film covers a wall in the presence of a second immiscible electrolyte. Examples are electrochemical setups utilizing electrodes covered by a thin liquid film [10-12], for which the rate of electrode reactions is dictated by the EDL structure at the electrode surface (see [13] and references therein).

We shall start from the free-energy description of the system, expressing the free energy $F$ as

$$
F=\int f\left[\psi, n_{ \pm}\right] d^{3} \mathbf{r},
$$

where $f$ is the free-energy density expressed as (assuming monovalent ions and based on a mean-field approach)

$$
\begin{aligned}
f= & f_{b}-\frac{1}{2} \epsilon(\mathbf{r})(\nabla \psi)^{2}+\left(n_{+}-n_{-}\right) e \psi \\
& +k_{B} T\left\{n_{+}\left[\ln \left(\frac{n_{+}}{n_{\infty}}\right)-1\right]\right. \\
& \left.+n_{-}\left[\ln \left(\frac{n_{-}}{n_{\infty}}\right)-1\right]\right\} \\
& +\beta_{+}(\mathbf{r}) n_{+}+\beta_{-}(\mathbf{r}) n_{-}+\text {const. }
\end{aligned}
$$

Here $f_{b}$ is the free-energy density of the solvent, $k_{B} T$ is the thermal energy, $\psi$ is the electrostatic potential, $e$ is the electric charge, $\epsilon$ is the permittivity, $n_{\infty}$ is the bulk ionic number density, $n_{ \pm}$are ionic number densities, and $\beta_{ \pm}$are the ionic interaction energies with the solvent.

As the system consists of immiscible liquids of a known spatial arrangement, $f_{b}$ is known beforehand. Thus the equilibrium conditions can be obtained by employing $\delta F / \delta \psi=0$ and $\delta F / \delta n_{ \pm}=0$, i.e.,

$$
\frac{\delta F}{\delta \psi}=\frac{d}{d y}\left[\epsilon(y) \frac{d \psi}{d y}\right]+e\left(n_{+}-n_{-}\right)=0
$$

and

$$
\frac{\delta F}{\delta n_{ \pm}}= \pm e \psi+k_{B} T \ln \left(\frac{n_{ \pm}}{n_{\infty}}\right)+\beta_{ \pm}(y)=0 .
$$


Potential $\zeta_{2}$

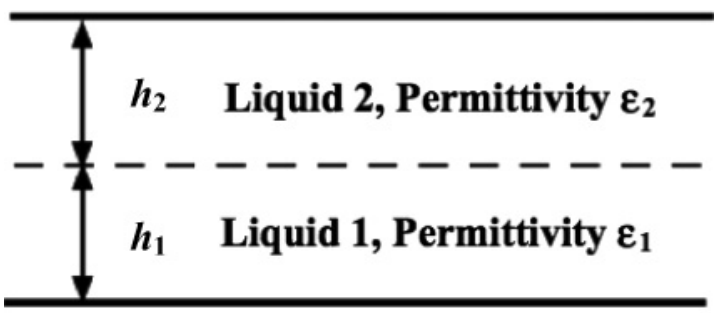

Potential $\zeta_{1}$

FIG. 1. Schematic of the problem.

Equation (4) gives the Boltzmann distribution as

$$
n_{ \pm}=n_{\infty} \exp \left(\frac{\mp e \psi-\beta_{ \pm}(y)}{k_{B} T}\right),
$$

demonstrating that one recovers the classical Boltzmann distribution in case $\beta_{ \pm}=0$.

Using Eq. (5) in Eq. (3), we get the Poisson-Boltzmann equation as

$$
\begin{aligned}
\frac{d}{d y}\left[\epsilon(y) \frac{d \psi}{d y}\right]= & e n_{\infty}\left[\exp \left(\frac{e \psi-\beta_{-}(y)}{k_{B} T}\right)\right. \\
& \left.-\exp \left(\frac{-e \psi-\beta_{+}(y)}{k_{B} T}\right)\right]
\end{aligned}
$$

We first consider the geometry as illustrated in Fig. 1, and assume the length of the lower layer $h_{1}$ as the relevant length scale. Hence the important dimensionless parameters are $\bar{\psi}=$ $e \psi / k_{B} T, \bar{y}=y / h_{1}, \bar{\beta}_{ \pm}=\beta_{ \pm} / k_{B} T$, and $\bar{\epsilon}=\epsilon /\left(\epsilon_{0} \epsilon_{w}\right)\left(\epsilon_{w}\right.$ is the relative permittivity of water), so that the dimensionless form of Eq. (6) is

$$
\begin{aligned}
\frac{d}{d \bar{y}}\left[\bar{\epsilon}(\bar{y}) \frac{d \bar{\psi}}{d \bar{y}}\right]= & \frac{1}{2} \frac{h_{1}^{2}}{\lambda^{2}}\left\{\exp \left[\bar{\psi}-\bar{\beta}_{-}(y)\right]\right. \\
& \left.-\exp \left[-\bar{\psi}-\bar{\beta}_{+}(y)\right]\right\},
\end{aligned}
$$

where $\lambda$ is the characteristic EDL thickness expressed as $\lambda=\sqrt{\epsilon_{0} \epsilon_{w} k_{B} T / 2 n_{\infty} e^{2}}$.

As the system consists of two immiscible liquid layers, we can express the permittivity and the ionic interaction potential profiles as

$$
\bar{\epsilon}=\bar{\epsilon}_{1} \theta(1-\bar{y})+\bar{\epsilon}_{2} \theta(\bar{y}-1)
$$

and

$$
\bar{\beta}_{ \pm}=\bar{\beta}_{1, \pm} \theta(1-\bar{y})+\bar{\beta}_{2, \pm} \theta(\bar{y}-1),
$$

where $\theta(x)$ is the Heaviside function.

Using Eqs. (8) and (9), we can simplify Eq. (7) as [we denote $\bar{\psi}$ for $0 \leqslant \bar{y} \leqslant 1$ as $\bar{\psi}_{1}$ and $\bar{\psi}$ for $1 \leqslant \bar{y} \leqslant\left(1+h_{2} / h_{1}\right)$ as $\left.\bar{\psi}_{2}\right]$

$$
\begin{aligned}
\bar{\epsilon}_{1} \frac{d^{2} \bar{\psi}_{1}}{d \bar{y}^{2}}= & \frac{1}{2} \frac{h_{1}^{2}}{\lambda^{2}}\left[\exp \left(\bar{\psi}_{1}-\bar{\beta}_{1,-}\right)-\exp \left(-\bar{\psi}_{1}-\bar{\beta}_{1,+}\right)\right] \\
& (\text { for } \quad 0 \leqslant \bar{y} \leqslant 1)
\end{aligned}
$$

and

$$
\begin{aligned}
\bar{\epsilon}_{2} \frac{d^{2} \bar{\psi}_{2}}{d \bar{y}^{2}}= & \frac{1}{2} \frac{h_{1}^{2}}{\lambda^{2}}\left[\exp \left(\bar{\psi}_{2}-\bar{\beta}_{2,-}\right)-\exp \left(-\bar{\psi}_{2}-\bar{\beta}_{2,+}\right)\right] \\
& \left(\text { for } \quad 1 \leqslant \bar{y} \leqslant 1+\frac{h_{2}}{h_{1}}\right) .
\end{aligned}
$$

In the Debye-Hückel approximation, the above two equations can be analytically solved as

$$
\begin{aligned}
& \bar{\psi}_{1}= K_{1} \exp \left(\frac{h_{1}}{\lambda_{1}^{e}} \bar{y}\right)+K_{2} \exp \left(-\frac{h_{1}}{\lambda_{1}^{e}} \bar{y}\right) \\
&- \frac{\exp \left(-\bar{\beta}_{1,-}\right)-\exp \left(-\bar{\beta}_{1,+}\right)}{\exp \left(-\bar{\beta}_{1,-}\right)+\exp \left(-\bar{\beta}_{1,+}\right)} \\
&(\text { for } 0 \leqslant \bar{y} \leqslant 1)
\end{aligned}
$$

and

$$
\begin{gathered}
\bar{\psi}_{2}=K_{3} \exp \left(\frac{h_{1}}{\lambda_{2}^{e}} \bar{y}\right)+K_{4} \exp \left(-\frac{h_{1}}{\lambda_{2}^{e}} \bar{y}\right) \\
-\frac{\exp \left(-\bar{\beta}_{2,-}\right)-\exp \left(-\bar{\beta}_{2,+}\right)}{\exp \left(-\bar{\beta}_{2,-}\right)+\exp \left(-\bar{\beta}_{2,+}\right)} \\
\left(\text { for } 1 \leqslant \bar{y} \leqslant 1+\frac{h_{2}}{h_{1}}\right)
\end{gathered}
$$

where $\lambda_{1}^{e}=\lambda \sqrt{\frac{2 \bar{\epsilon}_{1}}{\exp \left(-\bar{\beta}_{1,-}\right)+\exp \left(-\bar{\beta}_{1,+}\right)}}, \lambda_{2}^{e}=\lambda \sqrt{\frac{2 \bar{\epsilon}_{2}}{\exp \left(-\bar{\beta}_{2,-}\right)+\exp \left(-\bar{\beta}_{2,+}\right)}}$ and $K_{1}, K_{2}, K_{3}$, and $K_{4}$ are constants.

These constants can be evaluated in the presence of the following boundary conditions:

$$
\begin{aligned}
\left(\bar{\psi}_{1}\right)_{\bar{y}=0} & =\bar{\zeta}_{1}, \quad\left(\bar{\psi}_{1}\right)_{\bar{y}=1}=\bar{\psi}_{c}, \quad\left(\bar{\psi}_{2}\right)_{\bar{y}=1}=\bar{\psi}_{c}, \\
\left(\bar{\psi}_{2}\right)_{\bar{y}=1+\frac{h_{2}}{h_{1}}} & =\bar{\zeta}_{2}, \quad\left(\bar{\epsilon}_{1} \frac{d \bar{\psi}_{1}}{d \bar{y}}\right)_{\bar{y}=1}=\left(\bar{\epsilon}_{2} \frac{d \bar{\psi}_{2}}{d \bar{y}}\right)_{\bar{y}=1}
\end{aligned}
$$

(here $\bar{\psi}_{c}$ will be determined a posteriori), so as to obtain

$\bar{\psi}_{1}=\left(B_{1}+\bar{\psi}_{c}\right) \frac{\sinh \left(\frac{h_{1}}{\lambda_{1}^{e}}\right)}{\sinh \left(\frac{h_{1}}{\lambda_{1}^{e}}\right)}+\left(B_{1}+\bar{\zeta}_{1}\right) \frac{\sinh \left(\frac{h_{1}}{\lambda_{1}^{e}}(1-\bar{y})\right)}{\sinh \left(\frac{h_{1}}{\lambda_{1}^{e}}\right)}-B_{1}$

and

$$
\begin{aligned}
\bar{\psi}_{2}= & \left(B_{2}+\bar{\psi}_{c}\right) \frac{\sinh \left(\frac{h_{2}}{\lambda_{2}^{e}}+\frac{h_{1}}{\lambda_{2}^{e}}(1-\bar{y})\right)}{\sinh \left(\frac{h_{2}}{\lambda_{2}^{e}}\right)} \\
& +\left(B_{2}+\bar{\zeta}_{2}\right) \frac{\sinh \left(\frac{h_{1}}{\lambda_{2}^{e}}(\bar{y}-1)\right)}{\sinh \left(\frac{h_{2}}{\lambda_{2}^{e}}\right)}-B_{2},
\end{aligned}
$$

where $\quad B_{1}=\frac{\exp \left(-\bar{\beta}_{1,-}\right)-\exp \left(-\bar{\beta}_{1,+}\right)}{\exp \left(-\bar{\beta}_{1,-}\right)+\exp \left(-\bar{\beta}_{1,+}\right)} \approx \frac{\bar{\beta}_{1,+}-\bar{\beta}_{1,-}}{2-\bar{\beta}_{1,+}-\bar{\beta}_{1,-}}, \quad B_{2}=$ $\frac{\exp \left(-\bar{\beta}_{2,-}\right)-\exp \left(-\bar{\beta}_{2,+}\right)}{\exp \left(-\bar{\beta}_{2,-}\right)+\exp \left(-\bar{\beta}_{2,+}\right)} \approx \frac{\bar{\beta}_{2,+}-\bar{\beta}_{2,-}}{2-\bar{\beta}_{2,+}-\bar{\beta}_{2,-}}$ (here we use the condition, to be demonstrated later, that our analytical results are mostly valid for $\left|\bar{\beta}_{1 / 2, \pm}\right|<1$ ), and

$$
\begin{aligned}
\bar{\psi}_{c}= & \frac{\bar{\epsilon}_{1}\left[\frac{B_{1}+\bar{\zeta}_{1}}{\sinh \left(h_{1} / \lambda_{1}^{e}\right)}-B_{1} \operatorname{coth}\left(h_{1} / \lambda_{1}^{e}\right)\right]}{\bar{\epsilon}_{1} \operatorname{coth}\left(h_{1} / \lambda_{1}^{e}\right)+\bar{\epsilon}_{2} \frac{\lambda_{1}^{e}}{\lambda_{2}^{e}} \operatorname{coth}\left(h_{2} / \lambda_{2}^{e}\right)} \\
& +\frac{\bar{\epsilon}_{2}\left[\frac{B_{2}+\bar{\zeta}_{2}}{\sinh \left(h_{2} / \lambda_{2}^{e}\right)}-B_{2} \operatorname{coth}\left(h_{2} / \lambda_{2}^{e}\right)\right]}{\bar{\epsilon}_{1} \frac{\lambda_{2}^{e}}{\lambda_{1}^{e}} \operatorname{coth}\left(h_{1} / \lambda_{1}^{e}\right)+\bar{\epsilon}_{2} \operatorname{coth}\left(h_{2} / \lambda_{2}^{e}\right)} .
\end{aligned}
$$



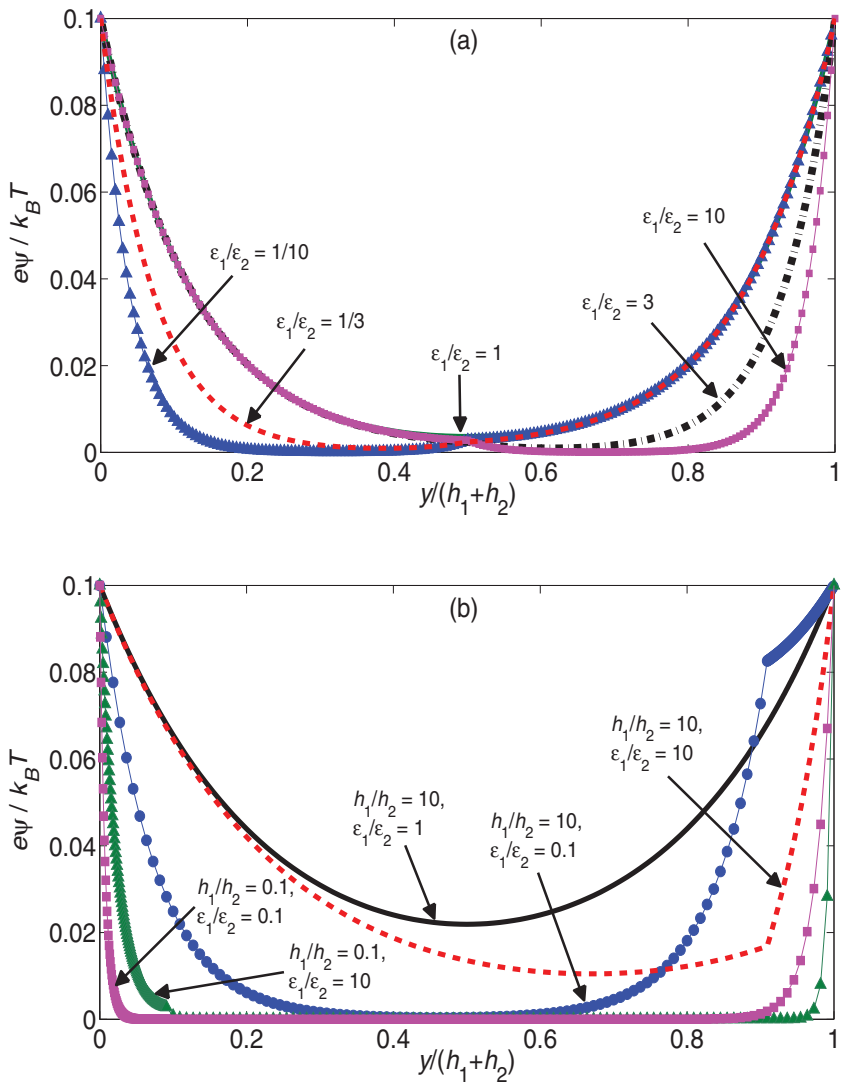

FIG. 2. (Color online) (a) Variation of the electrostatic potential for different values of permittivity ratios of the two fluids. In this plot $h_{1} / h_{2}=1, h_{1} / \lambda=4$, and $\bar{\zeta}_{1}=\bar{\zeta}_{2}=0.1$. (b) Variation of the electrostatic potential for different values of permittivity ratios as well as different values of the layer thickness of the two fluids. In this plot, $h_{1} / \lambda=4$ and $\bar{\zeta}_{1}=\bar{\zeta}_{2}=0.1$. In both of these plots $\bar{\beta}_{1,+}=$ $\bar{\beta}_{1,-}=\bar{\beta}_{2,+}=\bar{\beta}_{2,-}=0$.

The above results can be generalized for a system of $N$ immiscible electrolyte layers as

$$
\begin{aligned}
\bar{\psi}_{i}= & \left(B_{i}+\bar{\psi}_{c, i-1 / i}\right) \sinh \left[\frac{1}{\lambda_{i}^{e}} \sum_{k=1}^{i} h_{k}-\bar{y}\right] \\
& +\left(B_{i}+\bar{\psi}_{c, i / i+1}\right) \sinh \left[\bar{y}-\frac{1}{\lambda_{i}^{e}} \sum_{k=1, i>1}^{i-1} h_{k}\right]-B_{i}
\end{aligned}
$$$$
\text { (for } 1 \leqslant i \leqslant N \text { ), }
$$

where $\bar{\psi}_{c, i / j}$ is the potential at the interface between the adjacent layers $i$ and $j$. Note that the known potentials at the bottom and the top boundaries are denoted as $\bar{\psi}_{c, 0 / 1}$ and $\bar{\psi}_{c, N / N+1}$, respectively, while the unknown interface potential is

$$
\begin{aligned}
\bar{\psi}_{c, i / i+1}= & \frac{\bar{\epsilon}_{i}\left[\frac{B_{i}+\bar{\psi}_{c, i-1 / i}}{\sinh \left(h_{i} / \lambda_{i}^{e}\right)}-B_{i} \operatorname{coth}\left(h_{i} / \lambda_{i}^{e}\right)\right]}{\bar{\epsilon}_{i} \operatorname{coth}\left(h_{i} / \lambda_{i}^{e}\right)+\bar{\epsilon}_{i+1} \frac{\lambda_{i}^{e}}{\lambda_{i+1}^{e}} \operatorname{coth}\left(h_{i+1} / \lambda_{i+1}^{e}\right)} \\
+ & \frac{\bar{\epsilon}_{i+1}\left[\frac{B_{i+1}+\bar{\psi}_{c, i+1 / i+2}}{\sinh \left(h_{i+1} / \lambda_{i+1}^{e}\right)}-B_{i+1} \operatorname{coth}\left(h_{i+1} / \lambda_{i+1}^{e}\right)\right]}{\bar{\epsilon}_{i} \frac{\lambda_{i+1}^{e}}{\lambda_{i}^{e}} \operatorname{coth}\left(h_{i} / \lambda_{i}^{e}\right)+\bar{\epsilon}_{i+1} \operatorname{coth}\left(h_{i+1} / \lambda_{i+1}^{e}\right)} \\
& (\text { for } \quad 1 \leqslant i \leqslant N-1),
\end{aligned}
$$
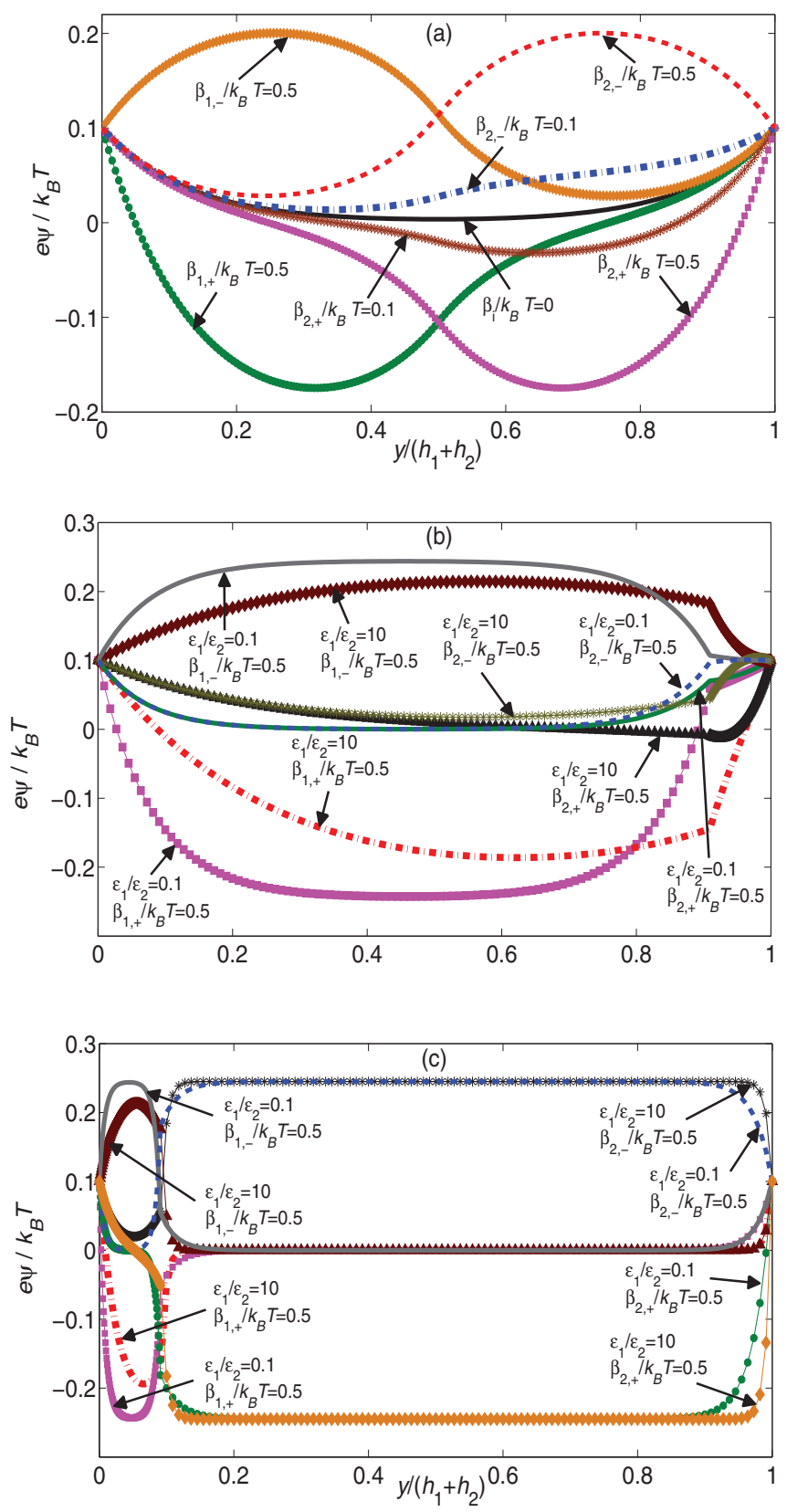

FIG. 3. (Color online) (a) Variation of the electrostatic potential for different values of the effective ionic potentials $\beta_{i}$. Other parameters are $\epsilon_{1} / \epsilon_{2}=1, h_{1} / h_{2}=1, h_{1} / \lambda=4$, and $\bar{\zeta}_{1}=\bar{\zeta}_{2}=0.1$. (b) Variation of the electrostatic potential for different values of effective ionic potentials $\beta_{i}$ 's and different values of permittivity ratios. Other parameters are $h_{1} / h_{2}=10, h_{1} / \lambda=4$, and $\bar{\zeta}_{1}=\bar{\zeta}_{2}=$ 0.1. (c) Repetition of the plots in (b), but with $h_{1} / h_{2}=1 / 10$. In all these plots, only that $\beta_{i}$ that has a finite nonzero value has been mentioned.

where $\lambda_{i}^{e}=\lambda \sqrt{\frac{2 \bar{\epsilon}_{i}}{\exp \left(-\bar{\beta}_{i,-}\right)+\exp \left(-\bar{\beta}_{i,+}\right)}} \approx \lambda \sqrt{\frac{2 \bar{\epsilon}_{i}}{2-\bar{\beta}_{i,+}-\bar{\beta}_{i,-}}}$ and $B_{i}=$ $\frac{\exp \left(-\bar{\beta}_{i,-}\right)-\exp \left(-\bar{\beta}_{i,+}\right)}{\exp \left(-\bar{\beta}_{i,-}\right)+\exp \left(-\bar{\beta}_{i,+}\right)} \approx \frac{\bar{\beta}_{i,+}-\bar{\beta}_{i,-}}{2-\bar{\beta}_{i,+}-\bar{\beta}_{i,-}}$ (here we use the condition, to be demonstrated later, that our analytical results are mostly valid for $\left.\left|\bar{\beta}_{i, \pm}\right|<1\right)$. 
From the known values of $\psi_{c, 0 / 1}$ and $\psi_{c, N / N+1}$, one can employ Eqs. (18) and (19) successively to obtain the potential distribution within any layer $i$ and the potential at the interface between any two adjacent layers $i$ and $i+1$. Henceforth, we shall provide results only corresponding to $N=2$; however, Eqs. (18) and (19) can always be invoked to provide results for any $N$.

We first study the electrostatic potential variations for the case of no ion-solvent interaction potentials [see Figs. 2(a) and 2(b)]. A smaller permittivity for a given liquid implies that the corresponding potential gradient must be steeper, as is evident from Figs. 2(a) and 2(b). For $h_{1} / h_{2}>1$, the relative EDL thickness (with respect to the entire channel height) is enhanced, whereas for $h_{1} / h_{2}<1$, it is diminished [see Fig. 2(b)].

Figures 3(a)-3(c) represent the central results of this Brief Report, demonstrating the effect of finite ion-solvent interaction energies. As a consequence, the overall electrostatic potential in the system will result from the mutually augmenting and nullifying effects of the EDLs at the boundaries and those induced by the jumps in ion-solvent interaction potential at the interfaces between the layers. We first consider the case of layers with identical permittivities and thicknesses [Fig. 3(a)]. A jump for the anions (i.e., a finite positive $\beta_{1,-}$ or $\beta_{2,-}$ ) lowers the overall anion concentration in the system [see Eq. (5)]. For example, in the case of a finite positive $\beta_{1,-}$, the lower electrolyte layer acquires a net positive charge. For large enough values of $\beta_{1,-}$, instead of screening the wall charge, this charge has the opposite effect of letting the electrostatic potential increase over the value prescribed at the wall. By contrast, a finite positive $\beta_{1,+}$ lowers the overall cation concentration in the system and brings additional negative charges into the lower layer, leading to a fast screening of the wall potential and large negative potential values.

Figures 3(b) and 3(c) show the coupled effects of finite interfacial ion-solvent interaction energy jumps as well as differences in the permittivity and the layer thickness of the two liquids. As has been discussed before, $h_{1} / h_{2}>1$ implies a more dominant influence of the wall EDL (since the EDL thickness has been fixed relative to $h_{1}$ ), whereas $h_{1} / h_{2}<1$ signifies the reverse. Also, such a variation in the thicknesses of the individual layers will imply unequal effects of the jumps in ionic energies $\beta_{1, \pm}$ or $\beta_{2, \pm}$. For $h_{1} / h_{2}>1$ [see Fig. 3(b)], a jump in $\beta_{2, \pm}$ has a much less pronounced effect. The effect is further decreased in case $\epsilon_{1} / \epsilon_{2}<1$. On the contrary, jumps in $\beta_{1, \pm}$ have a remarkable influence on the overall potential. Depending on whether the jump amplifies (caused by a jump in $\beta_{1,-}$ ) or reverses (caused by a jump in $\beta_{1,+}$ ) the boundary effect, one gets either an almost uniformly enhanced (as compared to the boundary potential) potential across the entire domain or a substantially large (compared to the magnitude of the wall potential) negative potential across the major portions of the domain. For $h_{2} / h_{1}>1$, interfacial effects show a greater influence on the overall electrostatic potential in the system for the case where there is a jump in the ionic interaction potential $\beta_{2, \pm}$ [see Fig. 3(c)].

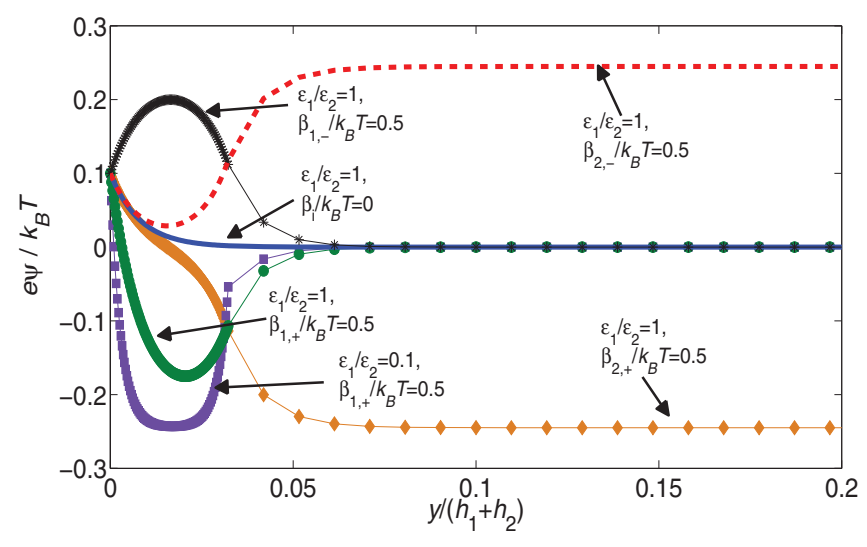

FIG. 4. (Color online) Variation of the electrostatic potential for the case when the top fluid layer is much thicker than the bottom one $\left(h_{2} \gg h_{1}\right.$; we take $\left.h_{2} / h_{1}=30\right)$. We provide results for different $\beta_{i}$ 's (only the $\beta_{i}$ that has a finite nonzero value is mentioned). Other parameters are $h_{1} / \lambda=4$ and $\bar{\zeta}_{1}=0.1$.

The present model can also be used to study an interesting limiting case, where an unbounded liquid medium is separated from a boundary by a very thin layer of immiscible electrolyte (i.e., we consider $h_{1} / h_{2} \ll 1$ ) (see Fig. 4). Most of the interesting effects occur within the thin electrolyte layer and, accordingly, we show results for a distance from the lower boundary up to $y /\left(h_{1}+h_{2}\right)=0.2$. These results can be easily interpreted from the results corresponding to Fig. 3(c). Figure 4 also allows us to verify whether, with the chosen parameters, the Debye-Hückel assumption is violated. One example is the case corresponding to $\epsilon_{1} / \epsilon_{2}=1$ and $\bar{\beta}_{2,+}=0.5$. For this case at large distances from the wall, one has $d^{2} \bar{\psi} / d \bar{y}^{2}=0$, implying that the resulting finite potential $\bar{\psi}_{0}$ must be obtained by equating the cation and anion concentrations, expressed in Eq. (5), and, without the Debye-Hückel approximation, this value is $\bar{\psi}_{0}=-\bar{\beta}_{2,+} / 2=-0.25$. From the plot, obtained under Debye-Hückel approximation, we recover $\bar{\psi}_{0}=-0.246$, implying that with the chosen parameters the results never violate the Debye-Hückel approximation. One can also apply Debye-Hückel linearization (to $\bar{\psi}$ ) to Eq. (5) and employ $\bar{\psi}=\bar{\psi}_{0}, \bar{\beta}_{-}=0$, and $\bar{\beta}_{+}=\bar{\beta}_{2,+}$ to obtain the condition $\bar{\psi}_{0}=1-2 /\left(1+e^{-\bar{\beta}_{2,+}}\right)$. Now if $\bar{\beta}_{2,+}$ is sufficiently small, such that $e^{\bar{\beta}_{2,+}} \approx 1-\bar{\beta}_{2,+}$ and $\left(1-\bar{\beta}_{2,+} / 2\right)^{-1} \approx 1+\bar{\beta}_{2,+} / 2$, we get back $\bar{\psi}_{0} \approx-\bar{\beta}_{2,+} / 2$. This establishes that one needs to operate at values of $\left|\bar{\beta}_{ \pm}\right|<1$ to ensure that our results never violate the Debye-Hückel approximation. In fact, $\left|\bar{\beta}_{ \pm}\right|<1$ ensures that the Debye-Hückel approximation is never violated in the bulk of the electrolyte, whereas $|\bar{\zeta}|<1$ (required to expand $\bar{\psi}$ in linearized form) ensures that it is never violated at the boundaries.

To summarize, we have demonstrated the remarkable impact of confining a system of immiscible electrolytes between two boundaries of given potential with a distance being of the order of the EDL thickness. We show that the net electrostatic potential in the system is dictated by the mutually supportive and opposing influences of two effects - the EDLs at the boundaries and the liquid-liquid interfacial ion-solvent interaction potential jump. 
[1] E. J. Verwey and K. F. Niessen, Philos. Mag. 28, 435 (1939).

[2] G. Gouy, J. Phys. 9, 457 (1910).

[3] D. L. Chapman, Philos. Mag. 25, 475 (1913).

[4] C. Gavach, P. Seta, and B. D'Epenoux, J. Electroanal. Chem. 83, 225 (1977).

[5] M. Gros, S. Gromb, and C. Gavach, J. Electroanal. Chem. 89, 29 (1978).

[6] A. Onuki, Phys. Rev. E 73, 021506 (2006).

[7] C. W. Monroe, M. Urbakh, and A. A. Kornyshev, J. Electroanal. Chem. 582, 28 (2005).
[8] I. Benjamin, Annu. Rev. Phys. Chem. 48, 407 (1997).

[9] Y. Tsori and L. Leibler, Proc. Natl. Acad. Sci. USA 104, 7348 (2007).

[10] A. L. Barker and P. R. Unwin, J. Phys. Chem. B 104, 2330 (2000).

[11] F. Quentel, V. Mirceski, and M. L'Her, Anal. Chem. 77, 1940 (2005).

[12] A. Nassi, C. T. Ebelle, E. Njanja, and E. Ngameni, Electroanalysis 23, 424 (2011).

[13] M. van Soestbergen, P. M. Biesheuvel, and M. Z. Bazant, Phys. Rev. E 81, 021503 (2010). 